\title{
NONSEPARATED MANIFOLDS AND COMPLETELY UNSTABLE FLOWS
}

\author{
SUDHIR K. GOEL \\ University of Houston-Downtown \\ Houston, Texas 77002
}

(Received August 18, 1986)

ABSTRACT. We define an order structure on a nonseparated n-manifold. Here, a nonseparated manifold denotes any topological space that is locally Euclidean and has a countable basis; the usual Hausdorff separation property is not required. Our result is that an ordered nonseparated $n$-manifold $X$ can be realized as an ordered orbit space of a completely unstable continuous flow $\phi$ on a Hausdorff $(n+1)$ manifold $E$.

KEY WORDS AND PHRASES. Completely unstable flows, nonseparated manifolds, order structure, orbit space.

1980 AMS SUBJECT CLASSIFICATION CODE. 58F25, 58F18.

\section{INTRODUCTION}

Nonseparated manifolds arise in a very natural way in the study of ordinary differential equations and completely unstable flows. A topological space that is non-Hausdorff, locally Euclidean and has a countable basis is referred to as a nonseparated manifold. A flow $\phi$ on a manifold $E$ is said to be completely unstable if it has no nonwandering points. Such systems occur very naturally. For example, on $\mathbb{R}^{2}$ any continuous flow without equilibria is completely unstable, and the restriction of any flow to the complement of its set of nonwandering points is completely unstable. All open manifolds admit completely unstable flows.

Let $\phi: E \times \mathbb{R}^{1} \rightarrow E$ be a completely unstable $c^{0}$ flow on an $(n+1)$-manifold $E$. The orbit space of $\phi$ is the set $E / \phi$ of all orbits of $\phi$ with the quotient topology (the finest topology in which the natural projection $\pi: E \rightarrow E / \phi$ is continuous). If $\phi$ admits local cross-sections at every point of $E$ that are $n$-Euclidean, we say $\phi$ is locally trivial. It is known that if either $E$ and $\phi$ are $c^{1}$ or $n \leq 2$, then $\phi$ is locally trivial ([1],[2]). Moreover, if $\phi$ is locally trivial, completely unstable $c^{0}$ flow then $E / \phi$ is a nonseparated $n$-manifold. The ordered orbit space of $\phi$ is obtained from this non-separated manifold by imposing an additional structure that indicates the order in which the cross-sections of $\phi$ that correspond to the charts of $E / \phi$ are traversed by orbits of $\phi$ (precise definitions are given in [3]). We then have the following classification theorem which shows that completely unstable flows on manifolds can be classified completely in terms of their associated ordered orbit spaces (Theorem $3.1,[3]$ ). 
CLASSIFICATION THEOREM. If $\phi$ and $\phi^{\prime}$ are locally trivial, completely unstable $c^{0}$ flows on m-manifolds $M$ and $M^{\prime}$, respectively, then $(M, \phi)$ and $\left(M^{\prime}, \phi^{\prime}\right)$ are topologically equivalent if and only if $M / \phi$ is order isomorphic to $M^{\prime} / \phi^{\prime}$.

Our interest here is in the question of realization: What nonseparated manifolds can be realized as the ordered orbit space of a completely unstable $c^{0}$ flow on some Hausdorff manifold? Some restriction on the nonseparated manifold is undoubtedly necessary. However, it appears to be a difficult problem to characterize the realizable ones. We present a preliminary result in this direction in the present paper. We first define a restricted class of nicely ordered nonseparated manifolds. We then prove that these manifolds are all realizable.

REALIZATION THEOREM. If $X$ is nicely ordered, nonseparated $n$-manifold then $X$ can be realized as the ordered orbit space of a completely unstable continuous flow $(E, \phi)$, where $E$ is a Hausdorff $(n+1)$-manifold.

Essentially the same result, in the case $X$ is a one-dimensional simply connected variety and $E=\mathbb{R}^{2}$ is stated in Haefliger and Reeb [4]. It is also stated in Neumann [3] for one-dimensional manifold $X$.

In $\S 2$ below, we give most of the definitions and notation required in the proof of the realization theorem; the proof itself occupies $\S 3-\S 5$. Finally, in $\S 6$ we prove the following corollary.

COROLLARY. Let $X$ and $E$ be as in the realization theorem. If $\pi_{n}(X)=0$, then $\pi_{n}(E)=0$ for $n \geq 1$. Moreover, if $X$ is a one-dimensional simply connected nonseparated manifold, then $E$ is homeomorphic to $\mathbb{R}^{2}$.

2. PRELIMINARIES.

DEFINITIONS AND NOTATION. Throughout what follows, $E$ denotes a Hausdorff $(n+1)$-manifold, $\phi: E \times \mathbb{R}^{1} \rightarrow E$ denotes a continuous flow on $E$, and $X$ denotes a nonseparated n-manifold with a countable basis $\left(V_{i}, \Psi_{i}\right)$ where each $V_{i}$ is homeomorphic to $D^{n}$, the compact unit $n$-disk. A topological space is a nonseparated manifold if it is locally Euclidean and has a countable basis, the usual Hausdorff separation axiom is not assumed. For any set $S \subseteq E, T \subseteq \mathbb{R}^{1}, S \cdot T=\{\phi(x, t) \mid x \varepsilon S$, $\left.t_{\varepsilon} T\right\} ; x \cdot T=\{x\} \cdot T$; and for $x \in E$ and $t_{\varepsilon} I R^{1}, x \cdot t=\phi_{t}(x)=\phi(x, t)$. The orbit of $x \varepsilon E$ is the set $\gamma(x)=x \cdot \mathbb{R}^{1}$. The orbit space $E / \phi$ is the set of all orbits of $\phi$ with the quotient topology. Also, throughout what follows, for any set $A$ contained in a topological space, $\AA$ and $\bar{A}$ will denote the interior and the closure of $A$ respectively.

$A$ set $U \subseteq E$ is said to be wandering (with respect to $\phi$ ) if there exists $t_{0} \in \mathbb{R}^{1}$ such that $U \cdot t \cap U=0$ for each $t$ with $|t| \geq t_{0}$. A point $x_{\varepsilon} E$ is nonwandering if it has no wandering neighborhood. Equivalently, $x \in E$ is nonwandering if $x \in J^{+}(x)$, here $J^{+}(x)$ denotes the set of limits of sequences $\left\{x_{n} \cdot t_{n}\right\}$, where $\left\{x_{n}\right\}$ converges to $x$ and $t_{n}$ tends to $\infty$. The (closed $\phi$ - invariant) set of all nonwandering points of $\phi$ is denoted as $\Omega(\phi)$. A flow $\phi$ is said to be completely unstable if $\Omega(\phi)=\phi$. A cross-section of $\phi$ is a set $S \subseteq E$ for which the mapping $h: S \times \mathbb{R}{ }^{1} \in$ defined by $h(s, t)=s \cdot t$ is a homeomorphism of $S \times \mathbb{R}^{1}$ onto a subset of $E$. 


\section{STATEMENT OF THE REALIZATION THEOREM.}

In order to state our main result, we first need to define the following order structure.

DEFINITION 3.1. Let $X$ be a nonseparated $n$-manifold with a countable atlas $v_{i}, \psi_{i}>$ where each $v_{i}$ is homeomorphic to $D^{n}$ (the compact unit $n$-disk), and 0

$\left\{V_{i}\right\}_{i \geq 1}$ forms an open cover for $x$. We say that $x$ is nicely ordered if there exists a collection of continuous functions $h_{i j}: v_{i} \cap v_{j} \rightarrow\{-1,1\}$ satisfying:

(a) $h_{i j}(x)=-h_{j i}(x)$ for every $x \in V_{i} \cap v_{j}$;

(b) If $x \in V_{i} \cap v_{j} \cap v_{k}$ with $h_{i j}(x)=+1(-1)$ and $h_{j k}(x)=+1(-1)$, then $h_{i k}(x)=+1(-1)$;

(c) If $\left\{x^{n}\right\}$ is a sequence in $v_{j} \cap v_{j} \cap v_{k}(i<j<k)$ converging to $x \in V_{i}$, and $x \notin V_{j}$, then $x \notin V_{k}$.

The order structure defined as above is a generalization of the order structure on a nonseparated 1-manifold, as given by Neumann in [5]. However, the property (c) of the order structure as above is slightly more restrictive than the property (3) of the order structure given by Neumann (see $\$ 3.5$ below), and thus the phrase "nicely ordered" is used.

Our main result is the following Realization Theorem.

REALIZATION THEOREM. 3.2. If $X$ is a nicely ordered, nonseparated n-manifold, then $X$ can be realized as the ordered orbit space of a completely unstable continuous flow $(E, \phi)$, where $E$ is a Hausdorff $(n+1)$ - manifold.

REMARKS 3.3. ( $\alpha$ ) This result in the case $X$ is a one-dimensional simply connected variety and $E=\mathbb{R}^{2}$ is stated in Haefliger and Reeb [4]. It is also stated in Neumann [3] for one-dimensional manifold $X$.

( $B$ ) Properties (a) and (b) of the order structure defined in 3.1 above will be used implicitly throughout the proof of the realization theorem.

OUTLINE 3.4. We shall prove the realization theorem by induction on the number of charts in $X$ in the following two steps.

(1) We first show that $X$ can be realized as a base space of a bundle $B=\langle E, p, X\rangle$, where $E$ is a Hausdorff $(n+1)$ - manifold.

(2) We then define a flow $\phi$ on $E$, show that it is completely unstable and finally show that $X$ is the orbit space of the dynamical system $(E, \phi)$.

The first step, that is to show the existence of the bundle $B=\langle E, p, X\rangle$, is the major step in the proof of the realization theorem.

DISCUSSION 3.5. We would like to point out that the direct generalization of the order structure given by Neumann for nonseparated 1-manifold in [5] would be: (a) and (b) same as in the definition 3.1 above and replace (c) by a less restrictive condition $\left(c^{\prime}\right)$ as follows: 
(c') If $\left\{x^{n}\right\}$ is a sequence in $v_{j} \cap v_{j} \cap v_{k}$ such that

(a) $h_{i j}\left(x^{n}\right)=1$ and $h_{j k}\left(x^{n}\right)=1$ for each $n$, and

(B) $x^{n} \rightarrow x \in V_{i}$ with $x \notin V_{j}$, then $x \notin V_{k}$.

Moreover, if $\phi$ is a completely unstable $c^{0}$ flow on the $(n+1)$ - manifold $E$ and admits cross-sections that are locally Euclidean, then $E / \phi$ can be ordered in this sense: choose a covering system $\left\{S_{i}\right\}_{i \geq 1}$ of cross-sections for the dynamical system $(E, \phi)$ (see $4.2,4.3$ of $[3])$. Set $v_{i}=p\left(S_{i}\right)$ for each $i$. Then $\left\{\stackrel{\circ}{v}_{i}\right\}_{i \geq 1}$ forms an open cover of $E / \phi$. Let $f_{i j}: V_{j} \cap v_{j} \rightarrow \mathbb{R}^{1}$ be defined as in the proof of the classification theorem (Theorem 3.1, [3]). Set $h_{i j}(x)=\operatorname{sgn}\left(f_{i j}(x)\right), x \in v_{i} \cap v_{j}$. Using the properties of $f_{i j}$ (see [3]), it is now immediate that $h_{i j}$ satisfy the properties (a), (b) and ( $\left.c^{\prime}\right)$ above.

4. EXISTENCE OF A BUNDLE $B=\langle E, p, X\rangle$.

In the setting of the existence theorem (Theorem 3.2 of [6]), to show the existence of a bundle $B=\left\langle E, p, x>\right.$, we seek the coordinate transformations $\left\{g_{i j}\right\}$ in the space $X$, with the structure group the group $\tau$ of all translations of $\mathbb{R}^{1}$. In particular, we seek the maps:

$$
g_{i j}: v_{i} \cap v_{j} \rightarrow \tau \text { satisfying: }
$$

(a) $g_{i j}(x) \circ g_{j k}(x)=g_{i k}(x)$ for each $x \in v_{i} \cap v_{j} \cap v_{k}$ (compatibility condition);

(b) If $\left\{x^{n}\right\}$ is a sequence in $v_{j} \cap v_{j}$ such that $\left\{x^{n}\right\}$ converges to both $x_{i} \in v_{i}$ and $x_{j} \in v_{j}$ with $x_{i} \neq x_{j}$, and $h_{i j}\left(x^{n}\right)=+1(-1)$ for all $n$, then $g_{i j}\left(x^{n}\right)(t) \rightarrow+\infty(-\infty)$ as $n \rightarrow \infty$ (for every $\left.t \in \mathbb{R}^{1}\right)$.

We define $g_{i j}$ in terms of the translations $f_{i j}$ as follows:

$$
\begin{aligned}
g_{i j}: & v_{i} \cap v_{j} \rightarrow \tau \\
& x \rightarrow g_{i j}(x): \mathbb{R}^{1} \rightarrow \mathbb{R}^{1} \text { such that } \\
& g_{i j}(x)(t)=t+f_{i j}(x) ; x \varepsilon v_{i} \cap v_{j} \text { and } t \varepsilon \mathbb{R}^{1} .
\end{aligned}
$$

Where

$$
f_{i j}: v_{i} \cap v_{j} \rightarrow I R^{1}
$$

are to be defined so as to satisfy:

(A) $f_{i j}(x)+f_{j k}(x)=f_{i k}(x)$ for each $x \in v_{j} \cap v_{j} \cap v_{k}$; and

(B) If $\left\{x^{n}\right\}$ is a sequence in $x$ such that $\left\{x^{n}\right\}$ converges to both 


$$
\begin{aligned}
& x_{i} \varepsilon \stackrel{\circ}{V}_{i} \text { and } x_{j} \varepsilon \stackrel{\circ}{V}_{j} \text { with } x_{i} \neq x_{j} \text {, and } h_{i j}\left(x^{n}\right)=+1(-1) \text { for all } n \text {, then } \\
& f_{i j}\left(x^{n}\right) \rightarrow+\infty(-\infty) \text { as } n \rightarrow \infty .
\end{aligned}
$$

If we assume that $f_{i j}$ satisfying $(A)$ and $(B)$ exist, then $g_{i j}$ defined by $(*)$ trivially satisfy (b). For (a), fix $x \in v_{j} \cap v_{j} \cap v_{k}$ and $t \varepsilon \mathbb{R}^{1}$.

Then using $(A)$ for $f_{i j}$, we have

$$
\begin{aligned}
& g_{i j}(x) \circ g_{j k}(x)(t)=g_{i j}(x)\left(t+f_{j k}(x)\right)=t+f_{j k}(x)+f_{i j}(x)=t+f_{i k}(x)= \\
& g_{i k}(x)(t) \text { as desired. }
\end{aligned}
$$

Thus, to show the existence of the coordinate transformations $\left\{g_{i j}\right\}$, we need to show the existence of the translations $\left\{f_{i j}\right\}$ satisfying $(A)$ and $(B)$ above. We show the existence of $\left\{f_{i j}\right\}$ by induction on the number of charts in $x$. Note that since each chart is Hausdorff and $X$ is not, $X$ can not have a single chart.

REMARK 4.1. One should note that the existence theorem (Theorem 3.2 of [6]) not only gives the existence of a bundle $B=\langle E, p, X\rangle$ but also its uniqueness up to bundle equivalence.

NOTATION 4.2. In What follows, $B_{1}$ denotes the set of all non-Hausdorff points of $X$ and $V_{i j}(i \neq j)$ denotes the set of all those points $x_{i} \varepsilon B_{1} \cap v_{i}$ such that there exists a sequence $\left\{x^{n}\right\}$ in $v_{i} \cap v_{j}$ with $\left\{x^{n}\right\}$ converging to $x_{i}$ and also to another point $x_{j} \in V_{j}$ with $x_{i} \neq x_{j}$. Note that $V_{i j}$ is the set of all those non-Hausdorff points in $V_{i}$ that can not be separated from some point in $V_{j}$.

PROPOSITION 4.3 For any $i \neq j$, the set $v_{i j}$ is a closed subset of the metric space $V_{i}$.

PROOF: Let $\left\{y_{k}\right\}$ be a sequence in $v_{i j}$ such that $\left\{y_{k}\right\}$ converges to $y \varepsilon V_{i}$. We want to show that $y \varepsilon V_{i j}$. Without loss of generality, let $y_{k} \frac{\varepsilon_{B} \frac{1}{2^{k}}}{}(y)$ for each $k$,

where $\frac{B_{1}}{2^{k}}(y)$ is an open ball in the metric space $v_{i}$ Moreover, for each $k$, let $\left\{x_{k}^{n}\right\}$ be a sequence in $v_{j} \cap v_{j}$ such that $\left\{x_{k}^{n}\right\}$ converges to $y_{k}$ and also to another point $y_{k}^{\prime} \in V_{j}$, with $y_{k} \neq y_{k}^{\prime}$. Since $v_{j}$ is compact, the sequence $\left\{y_{k}^{\prime}\right\}$ has a convergent subsequence $\left\{y_{k_{\ell}^{\prime}}^{\prime}\right\} \rightarrow y^{\prime} \varepsilon V_{j}$. As above, let $y_{k_{\ell}}^{\prime} \frac{\varepsilon_{B}}{2^{l}}\left(y^{\prime}\right)$ for each $\ell$, where $\frac{B_{1}}{2^{l}}\left(y^{\prime}\right)$ is an open ball in $V_{j}$. By induction, there exists $N_{m}>N_{m-1}$ in $z^{+}$such that $x_{k_{m}}^{N_{m}} \varepsilon \frac{B_{1}}{2^{m}}(y) \cap \frac{B_{i}^{\prime}}{2^{m}}\left(y^{\prime}\right)$. Now the sequence $\left\{x_{k_{m}^{m}}^{N}\right\}$ is in $v_{i} \cap v_{j}$ and obviously

converges to both $y$ and $y^{\prime}$. Moreover, it can be easily seen that $y \neq y^{\prime}$. Hence $y \varepsilon V_{i j}$ as desired. 
EXISTENCE OF $f_{i j}$ FOR TWO CHARTS 4.4 . If $X$ has only two charts, say $v_{1}$ and $v_{2}$, then $\left\{f_{i j}\right\}(1 \leq i, j \leq 2)$ satisfying $(A)$ and $(B)$ above exist for these two charts.

Let $X^{\prime}=\left(V_{1} \cap V_{2}\right), V_{12}$ (disjoint union). Note that $X^{\prime}$ is a metric subspace of the metric space $V_{1}$. Define $f^{\prime}: X^{\prime} \rightarrow[0,1]$ by

$$
f^{\prime}(x)=\frac{1}{1+d\left(x, v_{12}\right)} ; x \in x^{\prime} .
$$

Then $f^{\prime}$ is a continuous function, and since $v_{12}$ is a closed set $(\$ 4.3), f^{\prime}(x)=1$ if and only if $x \in v_{12}$. Define $f_{12}: x^{\prime}+[0, \infty]$ by $f_{12}(x)=\tan \frac{\pi}{2}\left(f^{\prime}(x)\right)$. Then $f_{12}$ is continuous and $f_{12}^{\prime}(x)=\infty$ if and only if $x \in v_{12}$. Now set $f_{12}=f_{12} \mid v_{1} \cap v_{2}$, where $f_{12}^{\prime} \mid v_{1} \cap v_{2}$ indicates the restriction of the function $f_{12}$ on the set $v_{1} \cap v_{2}$.

Finally, the set $f_{12}, f_{21}=-f_{12}$, and $f_{i j}=O(i=1,2)$ is the desired set of $f_{i j}(1 \leq i, j \leq 2)$, satisfying $(A)$ and $(B)$ above. This completes the construction of $f_{i j}$ in the case $x$ has only two charts.

REMARK 4.5. In the construction of $f_{12}$ above, observe that $f_{12}(x)>0$ for every $x \in V_{1} i V_{2}$. In the rest of the proof, we would construct $f_{i j}$ so as to satisfy (A) and (B) above and also the following added property:

$$
\text { (C) If } j>i \text {, then } f_{i j}(x)>0 \text { for every } x \in v_{i} \cap v_{j} \text {. }
$$

INDUCTION STEP 4.6. Suppose that we can define $\left\{f_{i j}\right\}(1 \leq i, j \leq n)$ satisfying $(A),(B)$, and $(C)$ above in the case $x$ has $n$-charts say $v_{1}, v_{2}, v_{3}, \ldots, v_{n}$, we show that $\left\{f_{i j}\right\}$ satisfying $(A),(B)$, and $(C)$ above can be defined in the case $X$ has $(n+1)$ - charts $v_{1}, v_{2}, \ldots, v_{n}, v_{n+1}$.

In order to show the existence of $\left\{f_{i j}\right\}$ in the case $X$ has $(n+1)$ - charts, we first need to show the existence of $\left\{f_{i j}\right\}$ in the case $X$ has only three charts, which in turn requires the following lemma:

LEMMA 4.7. Let $A$ and $B$ be closed subsets of a metric space $Y$. If $g: A \rightarrow$ $[0,1]$ is a continuous map such that $g(x)=1$ if and only if $x \in A \cap B$, then $g$ can be extended to a continuous map $\bar{g}: Y \rightarrow[0,1]$ such that $\bar{g}(x)=1$ if and only if $x \in B$.

$$
\text { PRO0F: Define } \begin{aligned}
& g_{A \cup B}: A \cup B \rightarrow[0,1] \text { by } \\
& g(x) \text { if } x \in A ; \\
& g_{A \cup B}(x)= \begin{cases}\text { if } x_{\varepsilon} B .\end{cases}
\end{aligned}
$$

It is obvious that $g_{A} \cup B$ is a well-defined map that extends $g$. Also, it is continuous by glueing lemma ([7], page 50). Moreover, $g_{A \cup B}(x)=1$ if and only if $x \in B$.

In order to extend $g_{A \cup B}$ to the whole of $Y$, we observe that $A \cup B$ is a closed subspace of the metric space $Y$. Therefore, there exists a continuous function $u: Y \rightarrow[0,1]$ such that $u(x)=1$ if and only if $x \in A \cup B$. Moreover, by Tietze Extension Theorem, there exists a continuous extension $g^{\prime}: Y \rightarrow[0,1]$ of $g_{A} \cup B$ such that $g^{\prime}(x)=1$ if $x \in B$. 
Finally, define $\bar{g}: Y \rightarrow[0,1]$ by $\bar{g}(x)=u(x) \cdot g^{\prime}(x)$ for $x \varepsilon Y$. It can be easily seen that $\bar{g}$ is the desired map. This completes the proof of the lemma.

EXISTENCE OF $f_{i j}$ FOR THREE CHARTS 4.8. If $x$ has only three charts, say $v_{1}$, $v_{2}$, and $v_{3}$, then $\left\{f_{i j}\right\}(1 \leq i, j \leq 3)$ satisfying $(A),(B)$ and $(C)$ above can be defined for these three charts.

Let $f_{12}^{\prime}:\left(v_{1} \cap v_{2}\right) \cup v_{12} \rightarrow[0, \infty]$ be the function as obtained in the case of two charts (cf. $\S 4.4)$. Define $f_{23}^{\prime}:\left(v_{2} \cap v_{3}\right) \cup v_{23} \rightarrow[0, \infty]$ analogous to $f_{12}^{\prime}$. Here, $V_{23}$ is a set as defined in $\$ 4.2$.

In order to define $f_{13}^{\prime}:\left(v_{1} \cap v_{3}\right) \cup v_{13} \rightarrow[0, \infty]$, let $A_{123}=v_{1} \cap v_{2} \cap v_{3}$ and define $V_{123}$ to be the set of all those points $x_{1} \in B_{1} \cap V_{1}$, such that there exists a sequence $\left\{x^{n}\right\}$ in $A_{123}$ with $\left\{x^{n}\right\}$ converging to $x_{1}$ and also to another point $x_{3}$ in $v_{3}$ with $x_{1} \neq x_{3}$, ( $B_{1}$ is the set as defined in $\$ 4.2$ above). Observe that $v_{123} \subseteq v_{13}$. We claim:

THEOREM 4.9. If $x_{1} \in V_{123}$ then either $x_{1} \in V_{12}$ or $x_{1} \varepsilon V_{23}$.

PROOF: If $x_{1} \in V_{123}$ then there exists a sequence $\left\{x^{n}\right\}$ in $A_{123}$ such that $\left\{x^{n}\right\}$ converges to $x_{1}$ and also to another point $x_{3}$ in $v_{3}$ with $x_{1} \neq x_{3}$. Since $v_{2}$ is compact, $\left\{x^{n}\right\}$ has a convergent subsequence $\left\{x^{k}\right\} \rightarrow x_{2} \in V_{2}$. If $x_{2}=x_{1} \neq x_{3}$, then $x_{1}$ $\varepsilon V_{23}$, otherwise $x_{1} \in V_{12}$.

We now define $f_{13}: A_{123} \cup V_{123} \rightarrow[0, \infty]$ by

$$
f_{13}^{\prime}(x)=f_{12}^{\prime}(x)+f_{23}^{\prime}(x) ; x \in A_{123} \cup V_{123} \text {. }
$$

Then $f_{13}^{\prime}$ is a well-defined continuous map. Since both $f_{12}^{\prime}$ and $f_{23}^{\prime}$ are finite on $A_{123}$, it follows from 4.9 above that $f_{13}^{\prime}(x)=\infty$ if and only if $x \in V_{123}$. We want to extend $f_{13}^{\prime}$ continuously to $f_{13}^{\prime}:\left(v_{1} \cap v_{3}\right) \cup v_{13} \rightarrow[0, \infty]$ such that $f_{13}^{\prime}(x)=\infty$ if and only if $x \in v_{13}$. (Note that the extension of $f_{13}$ is also denoted as $f_{13}$ ).

In the setting of the lemma 4.7 above, we have $Y=\left(V_{1} \cap V_{3}\right) \cup V_{13}, A=A_{123}$ $U V_{123}$ and $B=V_{13}$. Assuming $A$ to be a closed subset (proved below) of $Y$, define $g: A+[0,1]$ by $g(x)=\frac{2}{\pi}$ arc $\tan \left(f_{13}(x)\right)$ where $f_{13}$ is defined by (4.2) above. Let $\bar{g}: Y \rightarrow[0,1]$ be an extension of $g$ as obtained in the lemma 4.7. Define $f_{13}^{\prime}: Y \rightarrow[0$, o] by $f_{13}^{\prime}=\tan \frac{\pi}{2}(\bar{g}(x))$. Note that $f_{13}^{\prime}(x)=\infty$ if and only if $x \in B=V_{13}$. To complete the definition of $f_{13}^{\prime}$, we still need to show:

THEOREM 4.10 The set $A=A_{123} \cup V_{123}$ is a closed subset of $Y=\left(V_{1} \cap V_{3}\right) \cup V_{13}$.

PROOF: As in proposition 4.3, it can be seen that $V_{123}$ is a closed subset of $Y$. Thus, to complete the proof, it suffices to show that the closure of $A_{123}$ in $Y$ is contained in $A$.

Let $\left\{x^{n}\right\}$ be a sequence in $A_{123}$ such that $\left\{x^{n}\right\} \rightarrow x_{1} \varepsilon Y$. Since $Y=$ $\left(v_{1} \cap v_{3}\right) \cup v_{13}$ (disjoint union), either $x_{1} \varepsilon v_{1} \cap v_{3}$ or $x_{1} \varepsilon v_{13}$. Let us first consider the case $x_{1} \in v_{1} \cap v_{3}$. Since $v_{2}$ is compact, $\left\{x^{n}\right\}$ has a convergent subsequence $\left\{x^{k}\right\} \rightarrow x_{2} \in V_{2}$. We claim that $x_{2} \varepsilon V_{1}$ and thus $x_{2}=x_{1}$. If not, then $x_{1} k$ 
$v_{2}$, Thus by property $(c)$ of the definition of order structure, we have $x_{1} \notin v_{3}$, a contradiction. Hence, in this case, $x_{1} \notin A_{123} \subseteq A$.

If $x_{1} \in V_{13}$, then $x_{1} \notin V_{3}$. Also, since $V_{3}$ is compact, therefore the sequence $\left\{x^{n}\right\}$ admits a convergent subsequence $\left\{x^{k}\right\} \rightarrow x_{3} \varepsilon V_{3}$. Hence $x_{1} \in V_{123} \subseteq A$, as desired.

Finally, $f_{13}=f_{13}^{\prime}\left|v_{1} \cap v_{3}, f_{12}=f_{12}^{\prime}\right| v_{1} \cap v_{2}, f_{23}=f_{23}^{\prime} \mid v_{2} v_{3}$, $f_{j i}=-f_{i j}(1 \leq i, j \leq 3)$ and $f_{i j}=0(i=1,2,3)$ is the desired set of $\left\{f_{i j}\right\}$ satisfying $(A),(B)$ and $(C)$ above. Here, $f_{i j}^{\prime} \mid v_{i} \cap v_{j}$ denotes the restriction of $f_{i j}^{\prime}$ on $v_{i} \cap v_{j}(1 \leq i, j \leq 3)$. This completes the construction of $f_{i j}$ in the case $x$ has three charts.

We now return to our induction step. We want to define $\left\{f_{i j}\right\}(1 \leq i, j \leq n+1)$ satisfying $(A),(B)$ and $(C)$ in the case $x$ has $(n+1)$ charts $v_{1}, v_{2}, \ldots V_{n+1}$, knowing that $\left\{f_{i j}\right\}(1 \leq i, j \leq n)$ satisfying $(A),(B)$ and $(C)$ have already been defined in the case $x$ has $n$ charts $v_{1}, v_{2}, \ldots, v_{n}$. For convenience sake, we will use the following notation in the rest of the proof.

NOTATION 4.11. Any extension of $f_{i j}^{\prime}$ will be denoted as $f_{i j}^{\prime}$. For any $i, j$ and $k, A_{i j k}$ denotes the set $v_{i} \cap v_{j} \cap v_{k}$, and $v_{i j k}$ denotes the set of all points $x_{i}$ in $B_{1} \cap V_{i}\left(B_{1}\right.$ is the set of all non-Hausdorff points in $\left.X\right)$ such that there exists a sequence $\left\{x^{n}\right\}$ in $A_{i j k}$ with $\left\{x^{n}\right\}$ converging to $x_{i}$ and also to another point $x_{k}$ in $v_{k}$ with $x_{i} \neq x_{k}$. Moreover, for any $i \neq j, A_{i j}$ denotes the set $v_{i} \cap v_{j}$.

REMARK 4.12. For any $i<j<k$, the set $A_{i j k} \cup V_{i j k}$ is a closed set in $A_{i k} \cup V_{i k}$ (cf. \$4.10) and it would be denoted as $B_{i j k}$. This remark would be used implicitly throughout what follows.

We now start defining $f_{i j}$ for $(n+1)$ charts. Define $f_{n n+1}^{\prime}: A_{n n+1} \cup V_{n n+1}$ $\rightarrow[0, \infty]$ as in the case of two charts (cf. \$4.4). Next, define $f_{n-1}^{\prime} n+1: B_{n-1} n n+1$ $\rightarrow[0, \infty]$ by

$$
f_{n-1}^{\prime} n+1(x)=f_{n-1}^{\prime} n(x)+f_{n n+1}^{\prime}(x) ; x \in B_{n-1} n n+1
$$

as in $\S 4.9$ for three charts. Here, $f_{n-1}^{\prime} n$ has been defined at the induction step. Using lemma 4.7 , extend $f_{n-1}^{\prime} n+1$ to $f_{n-1}^{\prime} n+1: A_{n-1} n+1 \cup V_{n-1} n+1 \rightarrow[0, \infty]$ as was done in the case of three charts. We next define the function $f_{n-2}^{\prime} n+1$ as follows. Define

$$
\begin{aligned}
& f_{n-2 n+1}^{\prime}(x)=f_{n-2}^{\prime}(x)+f_{n n+1}^{\prime}(x) ; \quad x \in B_{n-2 n n+1} \text {, and } \\
& f_{n-2 n+1}^{\prime}(x)=f_{n-2 n-1}^{\prime}(x)+f_{n-1}^{\prime} n+1 \\
&
\end{aligned}
$$

where $f_{n-2}^{\prime} n$ and $f_{n-2}^{\prime} n-1$ have been defined at the induction step and $f_{n-1}^{\prime} n+1$ is obtained above. Using the induction hypothesis and (4.3) above, it can be easily seen that $f_{n-2}^{\prime} n+1$ is well defined, that is, $f_{n-2}^{\prime} n+1$ defined by (4.4) coincides with $f_{n-2}^{\prime} n+1$ defined by (4.5) on the intersection $\left(B_{n-2} n n+1 \cap B_{n-2} n-1 n+1\right)$. Finally, using lemma 4.7 with $Y=A_{n-2} n+1 \cup V_{n-2} n+1, A=B_{n-2} n n+1 \cup_{n-2} n-1 n+1$, 
and $B=V_{n-2} n+1$, extend $f_{n-2}^{\prime} n+1$ to $f_{n-2 n+1}^{\prime}: A_{n-2 n+1} \cup V_{n-2 n+1}+[0, \infty]$ as was done in the case of three charts.

Continuing this process, we obtain $f_{n-3}^{\prime} n+1, f_{n-4}^{\prime} n+1, \ldots$, and $f_{2 n+1}^{\prime}$ inductively. Finally, define $f_{i n+1}^{\prime}$ as follows:

$$
\begin{array}{ll}
f_{1 n+1}^{\prime}=f_{1 n}^{\prime}+f_{n n+1}^{\prime} & \text { on } B_{1 n n+1}, \\
f_{1 n+1}^{\prime}=f_{1 n-1}^{\prime}+f_{n-1 n+1}^{\prime} \text { on } B_{1 n-1 n+1}, \\
\text { - } \\
f_{1 n+1}^{\prime}=f_{13}^{\prime}+f_{3 n+1}^{\prime} & \text { on } B_{13 n+1}, \text { and } \\
f_{1 n+1}^{\prime}=f_{12}^{\prime}+f_{2 n+1}^{\prime} & \text { on } B_{12 n+1},
\end{array}
$$

where $f_{i n+1}^{\prime}(i=2,3, \ldots, n)$ are the functions obtained above and $f_{1 j}^{\prime}(j=2,3$, $\ldots, n)$ are the functions that have been defined at the induction step. Using the induction hypothesis and the definition of the functions $f_{i n+1}^{\prime}(2 \leq i \leq n)$, it can be seen that $f_{1}^{\prime}{ }_{n+1}$ is well defined. Finally, using lemma 4.7 with $Y=A_{1}{ }_{n+1} v_{1} n+1$, $A=\bigcup_{i=2}^{n}\left(B_{1 i n+1}\right)$ and $B=v_{1 n+1}$, extend $f_{1 n+1}^{\prime}$ to $f_{1 n+1}^{\prime}: A_{1 n+1} \cup v_{1 n+1}+[0, \infty]$ as was done in the case of three charts.

We now let $f_{i j}=f_{i j}^{\prime} \mid v_{i} \cap v_{j}, f_{j i}=-f_{i j}$ and $f_{i j}=0$ for $i, j=1,2, \ldots, n+1$. We claim that the set $\left\{f_{i j}\right\}(1 \leq i, j \leq n+1)$ so obtained is the desired set of functions satisfying $(A),(B)$ and $(C)$. From the construction of $\left\{f_{i j}\right\}$ it is obvious that the functions $\left\{f_{i j}\right\}$ satisfy both $(B)$ and $(C)$. For $(A)$, we need to show that $f_{i j}(x)+f_{j k}(x)=f_{i k}(x)$ for each $x \varepsilon v_{i} \cap v_{j} \cap v_{k}$ and for any $i, j$ and $k$ where $1 \leq i, j, k \leq n+1$. In view of induction hypothesis, we only need to prove it in the case when one of the $i, j$ or $k$ is $n+1$.

If $i=n+1$, we need to show $f_{n+1} j(x)+f_{j k}(x)=f_{n+1} k(x)$. If $k>j$, then $f_{n+1}(x)+f_{j k}(x)=-f_{j n+1}(x)+f_{j k}(x)=-\left(f_{j k}(x)+f_{k n+1}(x)\right)+f_{j k}(x)=-f_{j k}(x)$ $-f_{k n+1}(x)+f_{j k}(x)=f_{n+1} k(x)$ as desired. If $j>k$, then $f_{n+1}(x)+f_{j k}(x)=$ $-f_{j n+1}(x)-f_{k j}(x)=-\left(f_{k j}(x)+f_{j n+1}(x)\right)=-f_{k n+1}(x)=f_{n+1} k(x)$ as desired. The cases when $j$ or $k$ equals $(n+1)$ are analogous.

This completes the induction step and hence the construction of $\left\{f_{i j}\right\}$ for $i$, $\mathrm{j} \geq 1$.

Hence, by the existence theorem (Theorem 3.2, [6]), we get a bundle $B=\langle E, p, X\rangle$ with the base space $X$ and the coordinate transformations $\left\{g_{\mathbf{i j}}\right\}$. Also, any two such bundles are equivalent. Moreover, since $X$ is an $n$-manifold, $E$ is an $(n+1)$ - manifold. We finally show that

THEOREM 4.13 E is a Hausdorff space

PROOF: If not, let $e$ and $e^{\prime}$ be two nonseparated points in $E$. We have $\left\{\mathfrak{V}_{k}\right\}$ covers $x$, and since for each $k$ there exists a homeomorphism $\Psi_{k}: V_{k} \times R^{1} \rightarrow^{-1}\left(V_{k}\right)$, 
([6], page 7), each $p^{-1}\left(V_{k}\right)$ is Hausdorff. Consequently, there exists $j>i$, such that $\mathrm{e} \varepsilon \mathrm{p}^{-1}\left(\mathrm{v}_{j}\right)$ and $\mathrm{e}^{1} \varepsilon \mathrm{p}^{-1}\left(\mathrm{v}_{j}\right)$ with $\mathrm{p}^{-1}\left(\mathrm{v}_{j}\right) \cap \mathrm{p}^{-1}\left(\mathrm{v}_{j}\right) \neq \emptyset$. Moreover, there exist $x \in \dot{V}_{1}, x^{\prime} \varepsilon \dot{V}_{j}$ and $t, t^{\prime} \varepsilon \mathbb{P}^{1}$, such that $\psi_{j}(x, t)=e$ and $\psi_{j}\left(x^{\prime}, t^{\prime}\right)=e^{\prime}$. Let $\left\{V_{i}^{n}\right\}_{n \geq 1}$ and $\left\{V_{j}^{n}\right\}_{n \geq 1}$ be neighborhood systems at e and $e^{\prime}$, respectively, with $v_{i}^{n} \subseteq$ $p^{-1}\left(\mathcal{V}_{i}\right)$ and $v_{j}^{n} \subseteq p^{-1}\left(v_{j}\right)$ for all $n$. Since e and $e^{\prime}$ are nonseparated points, therefore, for each $n$, there exists $y_{n} \varepsilon v_{i}^{n} \cap v_{j}^{n}$. Let $x_{n}=p\left(y_{n}\right) \varepsilon v_{j} \cap v_{j}$ for each $n$. Then there exists $t_{n} \in \mathbb{R}^{1}$ such that $y_{n}=\psi_{i}\left(x_{n}, t_{n}\right)=\psi_{j}\left(x_{n}, g_{j j}\left(x_{n}\right)\left(t_{n}\right)\right)$ for each $n$, where $g_{i j}$ are the coordinate transformations as constructed above.

Since $y_{n}$ converges to both $e=\psi_{i}(x, t)$ and $e^{\prime}=\psi_{j}\left(x^{\prime}, t^{\prime}\right)$ and both $\psi_{i}$ and $\psi_{j}$ are homeomorphisms, it can be easily seen that $x_{n}$ converges to both $x$ and $x^{\prime}$; $t_{n} \rightarrow t$; and $g_{j i}\left(x_{n}\right)\left(t_{n}\right) \rightarrow t^{\prime}$ as $n \rightarrow \infty$. Since $t_{n} \rightarrow t$, therefore there exists $t_{0} \in \mathbb{R}^{1}$ such that $t_{n} \leq t_{0}$ for all $n$. Consequently

$$
g_{j i}\left(x_{n}\right)\left(t_{n}\right) \leq g_{j i}\left(x_{n}\right)\left(t_{0}\right) \text { for all } n \text {. }
$$

But from our construction of $g_{i j}$, we have that for any $t \in \mathbb{R}^{1}, g_{j j}\left(x_{n}\right)(t) \rightarrow-\infty$ as $n$ $\rightarrow \infty$ (because $j>i$ ). Thus, $g_{j i}\left(x_{n}\right)\left(t_{0}\right) \rightarrow-\infty$ and consequently $g_{j i}\left(x_{n}\right)\left(t_{n}\right) \rightarrow-\infty$ as $n \rightarrow \infty$. Also, $g_{j i}\left(x_{n}\right)\left(t_{n}\right) \rightarrow t^{\prime}$ (finite); a contradiction. Hence, $E$ is Hausdorff. This completes the proof of Step 1 .

5. $X$ AS AN ORDERED ORBIT SPACE.

We now show that we can define a completely unstable continuous flow $\phi$ on $E$ and that $X$ can be realized as an ordered orbit space of the dynamical system $(E, \phi)$, where $E$ is the Hausdorff manifold obtained in $\S 4$ above.

To define a flow $\phi: E \times \mathbb{R}^{1} \rightarrow E$. Fix $(q, s) \in E \times \mathbb{R}^{1}$. Since $\psi_{j}: V_{j} \times \mathbb{R}^{1} \rightarrow$ $p^{-1}\left(v_{j}\right)$ is a homeomorphism for each $j$ and $\left\{\dot{0}_{j}\right\}_{j \geq 1}$ cover $x$; therefore, there exists some $k \geq 1$ with $x \in \stackrel{\circ}{V}_{k}$ and $t \in \mathbb{R}^{1}$ such that $q=\psi_{k}(x, t)$. Define

$$
\phi(q, s)=\psi_{k}(x, t+s) \text {. }
$$

We first show that $\phi$ is well defined; that is, if $q$ also equals $\psi_{j}\left(x^{\prime}, t^{\prime}\right)$ for some $j \neq k, x^{\prime} \varepsilon V_{j}$ and $t^{\prime} \varepsilon \mathbb{R}^{1}$, then $\psi_{k}(x, t+s)=\psi_{j}\left(x^{\prime}, t^{\prime}+s\right)$.

Since $\psi_{k}(x, t)=q=\psi_{j}\left(x^{\prime}, t^{\prime}\right)=\psi_{k}\left(x^{\prime}, g_{k j}\left(x^{\prime}\right)\left(t^{\prime}\right)\right)$ and $\psi_{k}$ is a homeomorphism; therefore, $x=x^{\prime}$ and $g_{k j}\left(x^{\prime}\right)\left(t^{\prime}\right)=t$, and hence

as desired.

$$
\psi_{j}\left(x^{\prime}, t^{\prime}+s\right)=\psi_{k}\left(x^{\prime}, g_{k j}\left(x^{\prime}\right)\left(t^{\prime}+s\right)\right)=\psi_{k}\left(x^{\prime}, g_{k j}\left(x^{\prime}\right)\left(t^{\prime}\right)+s\right)=\psi_{k}(x, t+s)
$$

We next show that $\phi$ is a continuous flow. It is obvious that $\phi$ is a continuous function. Moreover, $\phi$ satisfies the group law for $\phi(q, 0)=\psi_{k}(x, t+0)=q$; and $\phi\left(\phi\left(q, s_{1}\right), s_{2}\right)=\phi\left(\psi_{k}\left(x, t+s_{1}\right), s_{2}\right)=\psi_{k}\left(x, t+s_{1}+s_{2}\right)=\phi\left(x, s_{1}+s_{2}\right)$.

We finally show that $\phi$ is completely unstable and that $x$ is the orbit space of the dynamical system $(E, \phi)$. To show that $\phi$ is completely unstable, fix $q \varepsilon E$. We want to show that $q$ admits a wandering neighborhood. Let $q=\psi_{k}\left(x, t_{0}\right)$ for some $x$ 
$\varepsilon V_{k}$ and $t_{0} \varepsilon \mathbb{R}^{1}$. Fix $\varepsilon>0$ and let $w_{j}=\psi_{k}\left(\dot{V}_{k},\left(t_{0}-\varepsilon, t_{0}+\varepsilon\right)\right)$. Then $w_{j}$ is the required wandering neighborhood of $q$ as $\left(w_{j} \cdot t\right) \cap w_{j}=\emptyset$ for all $t$ such that $|t|>2 \varepsilon$. In order to show that $x$ is an orbit space of $(E, \phi)$, fix $q \in E$. If $q=\psi_{k}(x, t)$ for some $x \in V_{k}$ and $t \in \mathbb{R}^{1}$, then for any $s \in \mathbb{R}^{1}$, we have $\phi(q, s)=\psi_{k}(x$, $t+s)$. Thus, $p(\phi(q, s))=p\left(\Psi_{k}(x, t+s)\right)=\left(p o \psi_{k}\right)(x, t+s)=x$.

Moreover, from the construction of the bundle $B=\langle E, p, x\rangle$ in the existence theorem (Theorem 3.2, [6]), it can be seen that the topology of $X$ as a base space is equivalent to the quotient topology. Thus, $X$ is the desired orbit space of the dynamical system ( $E, \phi)$.

As we have mentioned in the introduction, an ordered orbit space of $\phi$ can now be obtained from the orbit space $X$ by imposing an additional structure that indicates the order in which the cross-sections of $\phi$ that correspond to the charts of $x$, are traversed by orbits of $\phi$ (precise definition of the order structure is given in [3]).

Hence, $X$ can be realized as an ordered orbit space of a completely unstable $c^{0}$ flow on the Hausdorff $(n+1)$-manifold $E$. This completes the proof of the realization theorem.

6. COROLLARY.

Let $X$ and $E$ be as in the realization theorem. If $\pi_{n}(X)=0$, then $\pi_{n}(E)=0$ for $n \geq 1$. Moreover, if $X$ is one-dimensional simply connected nonseparated manifold, then $E$ is homeomorphic to $\mathbb{R}^{2}$.

PROOF. We consider the exact homotopy sequence

$\cdots \rightarrow \pi_{n}\left(\mathbb{R}^{1}\right)^{i_{\star}} \rightarrow \pi_{n}(E)^{P_{\star}} \rightarrow \pi_{n}(X) \stackrel{\Delta}{\rightarrow} \pi_{n-1}\left(\mathbb{R}^{1}\right) \rightarrow \cdots \pi_{2}(X) \stackrel{\Delta}{\rightarrow} \pi_{1}\left(\mathbb{R}_{\mathbb{R}}{ }^{1}\right)^{i_{\star}} \rightarrow \pi_{1}(E)^{P_{\star}}{ }^{\rightarrow}$ $\pi_{1}(X) \rightarrow$ - of the bundle $B=\left\langle E, p, X>\right.$. Since for each $n \geq 1, \pi_{n}(X)=0$ and $\pi_{n}\left(\mathbb{R}^{1}\right)=0$, it follows that $\pi_{n}(E)=0$ for each $n \geq 1$. In particular, $E$ is simply connected.

Now from the isomorphism theorem of Hurewicz ([6], page 91), we know that the first non-zero homology group and the first non-zero homotopy group have the same dimension and are isomorphic. Thus, we conclude that $H_{n}(E)=0$ for each $n \geq 1$, that is, $E$ is acyclic.

If $X$ is a one-dimensional simply connected nonseparated manifold, then from the proof of the Realization Theorem, $E$ is a two-dimensional Hausdorff manifold. Moreover, from above $E$ is simply connected. Therefore, $E$ is homeomorphic to $S^{2}$ or $\mathbb{R}^{2}$. But $S^{2}$ is not acyclic and hence $E$ is homeomorphic to $\mathbb{R}^{2}$.

ACKNOWLEDGEMENT. This research was supported by an Organized Research Grant from the University of Houston-Downtown. 


\section{REFERENCES}

1. WHITNEY, H. Regular families of curves, Ann. of Math. 34 (1933), 244-270.

2. WHITNEY, H. Cross-sections of curves in 3-space, Duke Math. J. 4 (1938), $222-226$.

3. GOEL, Sudhir K. and NEUMANN, Dean A. Completely Unstable Dynamical Systems, Trans. Amer. Math. Soc. 291 (1985), 639-668.

4. HAEFLIGER, and REEB, G. Variétés (non séparées) á une dimension et structures feuilletees du plan, Ens. Math. (2) $\underline{3}$ (1957), 107-125.

5. NEUMANN, Dean A. Completely Unstable Flows on Two Manifolds, Trans. Amer. Math. Soc. 225 (1977), 211-226.

6. STEENROD, N. The Topology of Fiber Bundles, Princeton University Press, 1951.

7. SINGER, I. M. and THORPE, J. A. Lecture Notes on Elementary Topology and Geometry, Springer-Verlag, New York, 1967. 


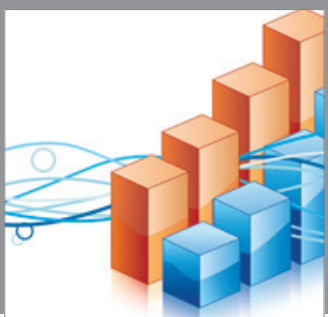

Advances in

Operations Research

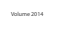

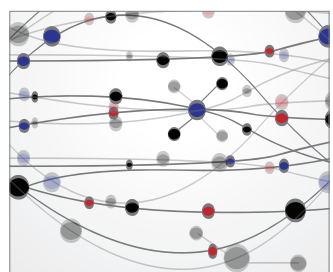

\section{The Scientific} World Journal
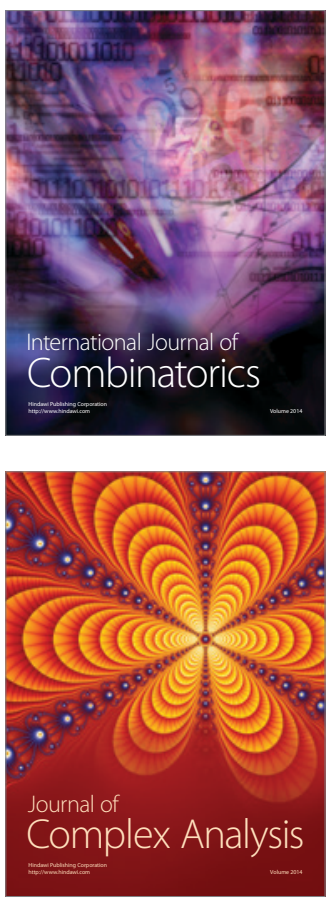

International Journal of

Mathematics and

Mathematical

Sciences
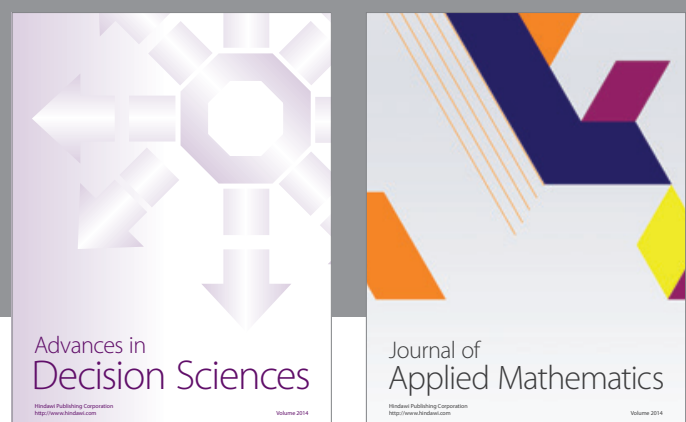

Journal of

Applied Mathematics
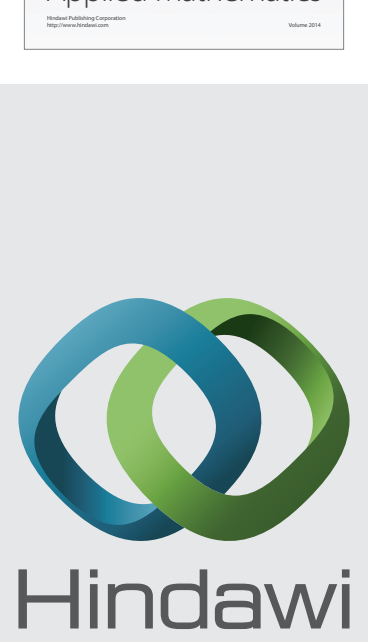

Submit your manuscripts at http://www.hindawi.com
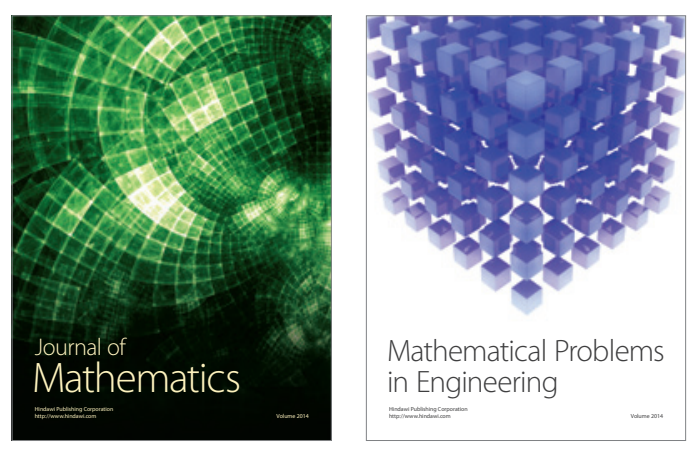

Mathematical Problems in Engineering
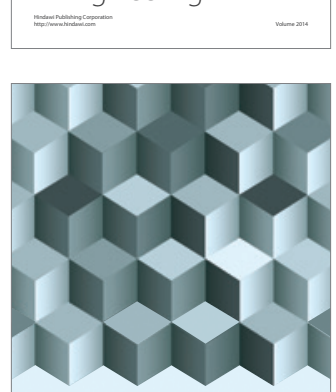

Journal of

Function Spaces
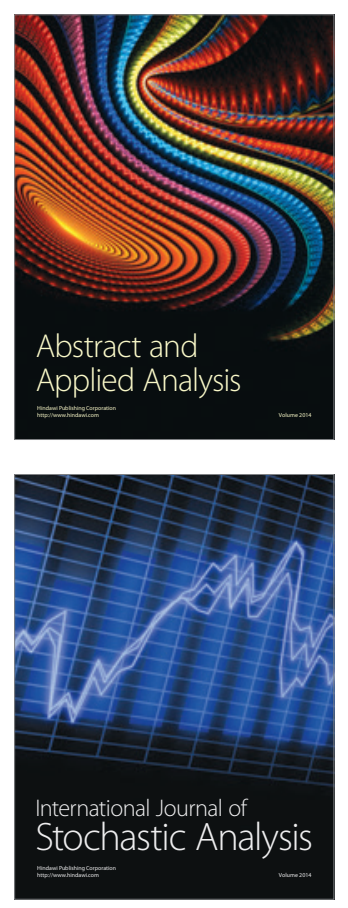

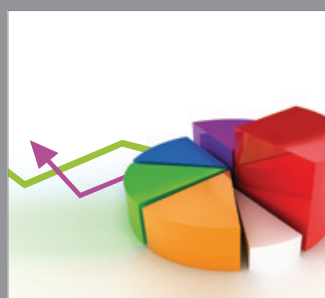

ournal of

Probability and Statistics

Promensencen
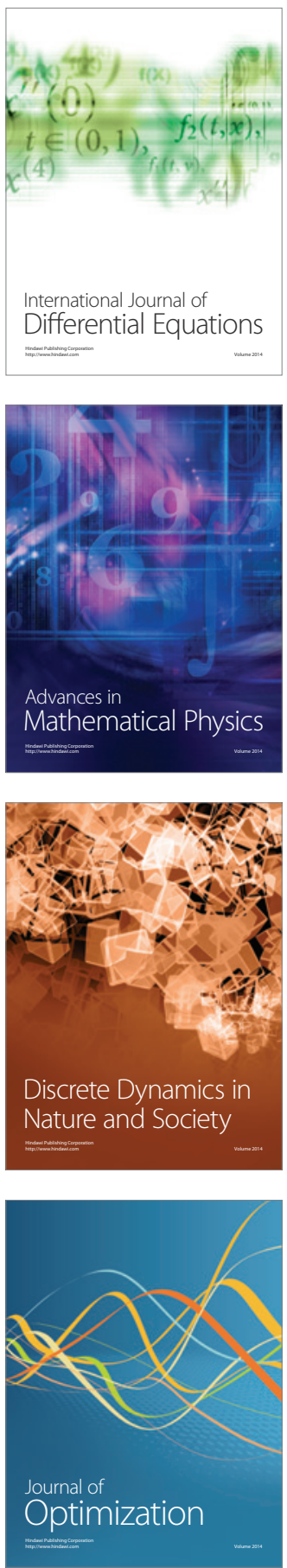